\title{
Pilot based Channel Estimation and SCGNN based Equalization for MIMO-OFDM System
}

\author{
Zeeshan A. Abbasi \\ Assistant Professor \\ University Polytechnic \\ Jamia Millia Islamia, New Delhi
}

\author{
Zainul Abdin Jaffery \\ Professor \\ Dept. of Electrical Engineering \\ Jamia Millia Islamia, New Delhi
}

\begin{abstract}
In this paper a new channel equalization technique is described based on Scaled Conjugate Gradient Neural Network (SCGNN) for Multiple Input Multiple Output (MIMO) Orthogonal Frequency Division Multiplexing (OFDM). To estimate the channel in frequency domain, Space Time Block Code (STBC) is used. At the beginning of each transmitted OFDM block the pilot sequence is inserted to estimate the channel. In the transmitter the 16-QAM modulation is used as it is efficient in conserving bandwidth. The performance is evaluated using MATLAB and various results are plotted at different values of SNR. It is observed from the simulation that the proposed channel estimation and equalization method greatly reduced the Symbol Error Rate. We are able to achieve the SER as low as 0.01at a very low value of SNR. The MMSE is also greatly reduced and the system converges very fast.
\end{abstract}

\section{General Terms}

ISI (Inter-symbol Interference), BER (Bit Error Rate), SNR (Signal to Noise Ratio)

\section{Keywords}

Scaled Conjugate Gradient Network, MIMO, OFDM, Equalization

\section{INTRODUCTION}

For wireless mobile communication the OFDM gained a lot of attention of the researchers as it fulfill the needs of high data transmission rates. OFDM gives huge channel capacity gain but the multipath effect is more severe as compare to the Single Input Single Output ( SISO ) system [1-4]. OFDM technology for wireless communication is becoming popular due to its advantages such as the high spectral efficiency, robustness to frequency selective fading and the feasibility of low cost transceiver implementations [5].OFDM is considered one of the most promising technology for present and future wireless communication used in $3 \mathrm{G}$ or WiMex [6].

To improve the performance of the OFDM system, channel estimation and equalization plays an important role. For OFDM over fast varying fading channels the channel estimation is carried out by transmitting known pilot symbols in given position. The equalization is to reduce the channel distortion causing Inter Symbol Interference (ISI). The circuit or equipment used to achieve equalization is called an equalizer. The equalizer strengthen or weaken the energy of specific frequency band [7]. In this paper the channel estimation is done using pilot based approach and equalization using SCGNN.

There are many pilot design techniques exist for I/Q imbalance estimation in Quadrature modulation.. The work in [8] explain the I/Q imbalance for SISO system. IT applies two OFDM training symbols per-subcarrier to perform estimation.
The first training symbol has null tones at all negative subcarrier indexes. The drawback of this method is that it neither optimizes the pilot overhead (large overhead).It also does not considers the complex problem of pilot-datamultiplexed symbols, MIMO-OFDM, and exploitation of frequency-domain correlation. Similar drawbacks apply to [9] with only the difference that it considers (mainly frequency independent (FI)) transmitter I/Q imbalance only, and uses a different pilot design. It uses an even number of OFDM training symbols with non-zero pilots where the pilots at the negative (positive) subcarrier indexes of the even symbols are the same as (negatives of) the corresponding pilots at the odd training symbols. The same pilot design using two OFDM training symbols is applied in [10] but one extra symbol is added for channel estimation for the FI receiver I/Q imbalance, resulting in a larger overhead. It considers pilotdata-multiplexed symbols, but the other drawbacks still hold. [11] applies a pilot design (a combination of the designs in [8] and [9] which uses an even number of OFDM training symbols with null pilots at the negative subcarrier indexes of the first half of OFDM training symbols and at the positive subcarrier indexes of the second half of OFDM training symbols. It considers FI receiver I/Q imbalance only, and is also associated with the above drawbacks. In this paper comb type pilot based channel estimation is used in which we have inserted $\mathrm{Np}$ pilot symbol in the series of $\mathrm{Z}(\mathrm{k})$ OFDM symbols as per the following equation:

$$
\mathrm{Z}(\mathrm{k})=\mathrm{Z}(\mathrm{mL}+\mathrm{l})
$$

Where $\mathrm{L}=$ number of sub carriers with respect to $\mathrm{N}_{\mathrm{p}}, \mathrm{m}$ is the pilot carrier index [12].

Several adaptive learning algorithms for feed-forward neural networks has already been discovered and implemented in equalizers. Many of these uses gradient decent algorithm but it suffers poor convergence rate and depends on parameters which have to be specified by the users because there is still no theoretical basis exist for choosing them. The value of these parameters are often crucial for the success of the algorithm e.g. back-propagation algorithm [13].The problem of optimizing the weight adjustment of neural network cab be effectively solve by Conjugate Gradient method.

\section{OFDM SYSTEM MODEL}

OFDM can be assumed as the special case of multicarrier wireless transmission. In OFDM a single data stream is transmitted over a number of low rate subcarriers. The idea of OFDM is to split the total bandwidth into a number of orthogonal subcarriers in order to transmit symbols using these sub carriers in parallel.

Figure 1 describe OFDM transmitter with two transmitting TX antenna and Figure 2 describe the OFDM receiver for two receive Rx antenna However, the idea can be applied to any number of $\mathrm{Tx}$ and $\mathrm{Rx}$ antennas. It can be seen from the 
figure 1 that pilots are inserted at the beginning of the data and then the data is modulated by any of the modulating scheme but here 16-QAM modulation is used as it is bandwidth conserving. The modulated data is then encoded using the space time encoder. The output of the encoder is then split into two path, one for each antenna as described for the simple case of MIMO space-time coding in [14]. From [14] as it is applied for the OFDM system, we can have the following vectors for TX antennas 1 and 2:

$T_{x_{1}}=\left[\begin{array}{llll}S_{1}-\widehat{S_{2}} & S_{3} & -\widehat{S_{4}} S_{N-1}-\widehat{S_{N}}\end{array}\right]$

$T_{x_{2}}=\left[\begin{array}{lllllll}S_{2} & \widehat{S_{1}} & S_{4} & \widehat{S_{3}} & \ldots & S_{N} & \widehat{S_{N-1}}\end{array}\right]$

where $T_{x_{1}}, T_{x_{2}}$ are the vectors of the output of two Tx

antennas 1 and 2 respectively.

$S_{1}, S_{2}$ are the pilot symbols and $S_{3}, S_{4}$ $S_{N}$ are the message symbols. The total symbols will be double of the number of subcarrier. The encoded data is then passed through the serial to parallel converter which regroup the data as per the table 1:

\begin{tabular}{|c|c|c|c|}
\hline Time & \multicolumn{3}{|c|}{ Carrier } \\
\hline $\mathrm{T}$ & 1 & 2 & $\mathrm{k}$ \\
\hline & $S_{1}$ & $S_{3}$ & $S_{N-1}$ \\
$\mathrm{t}+\mathrm{T}$ & $\widehat{S_{2}}$ & $-\widehat{S}_{4}$ & $\widehat{-S_{N}}$ \\
\hline
\end{tabular}

Before transmitting the signal to each antenna, a cyclic prefix is added to the message signal of each Tx antenna and then the signal is transmitted through the frequency selective channel. The cyclic prefix is assumed to be longer than the largest delay spread.The channel is assumed as the Rayleigh fading channel which is widely used as the channel in urban areas. Assuming $\mathrm{CH} 1$ and $\mathrm{CH} 2$ are the two channels from $\mathrm{Tx}$ antennas 1 and 2 respectively to the receiver.

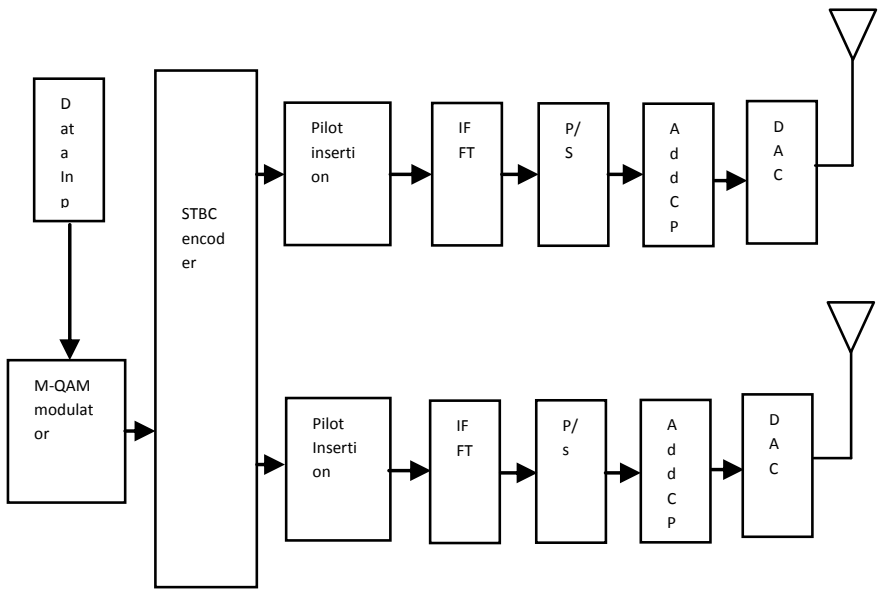

Figure 1. OFDM Transmitter with Two Transmitting Antennas

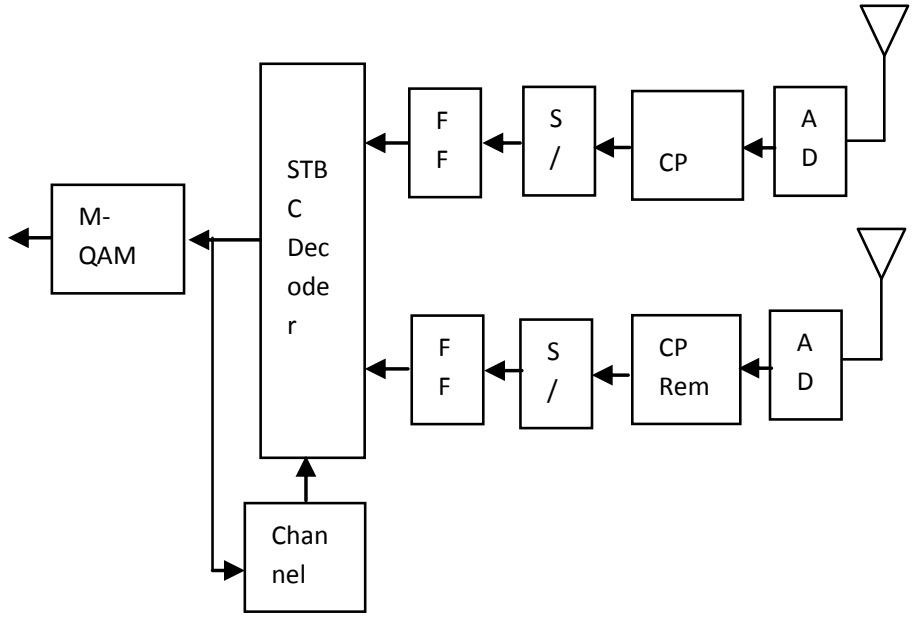

Figure 2. OFDM Receiver with Two Receiving Antennas

During transmission, noise also effect the transmitted signal and added to it thus by assuming $\mathrm{CH}[\mathrm{n}, \mathrm{k}]=\mathrm{CH}[\mathrm{n}+1, \mathrm{k}]$, the received signal can be expressed as:

$$
r[n, k]=C H_{1}[n, k] S_{1}[n, k]+C H_{2}[n, k] S_{2}[n, k]+\eta[n, k]
$$

where

$r[n, k]=$ Received Symbols

$S[n, k]=$ Transmitted Symbols

$\eta[n, k]=$ White Gaussian Noise (AWGN)

$[n, k]=\mathrm{n}$-th OFDM block and k-th sub-carrier

$S_{1}, S_{2}=$ symbols transmitted from the Tx antennas 1 and 2 respectively and are given by

$$
\begin{aligned}
& S_{1}=\left[\begin{array}{llllll}
s_{1} & s_{3} & s_{N-1} & -\hat{s}_{2} & -\hat{s}_{4} & -\hat{s}_{N}
\end{array}\right] \\
& S_{2}=\left[\begin{array}{llllll}
s_{2} & S_{4} & s_{N} & \hat{S}_{1} & \hat{S}_{3} & \hat{S}_{N-1}
\end{array}\right]
\end{aligned}
$$

Finally the received signal is decoded as given by [14] and the result is given by

$$
\begin{aligned}
& \hat{S_{i+1}}[n, k]=C H_{1}^{*}[n, k] r[n, k]+C H_{2}[n, k] r^{*}[n, k+1](6) \\
& \hat{S_{i+1}}[n, k]=C H_{2}^{*}[n, k] r[n, k]-C H_{1}[n, k] r^{*}[n, k+1]
\end{aligned}
$$

Where $i=1,3,5 \ldots . . N-1$ representing the symbol and $\hat{S}$ representing the decoded data

\section{PROPOSED CHANNEL ESTIMATION METHOD}

There are several techniques discussed in [15] to estimate the radio channel in OFDM system. In propose system we have 
used pilot aided channel estimation technique. In order to estimate the channel first a pilot symbol is transmitted. In OFDM system the pilot is transmitted in the beginning of each OFDM block. Once the channel is estimated, equation (6) \& (7) are used to decode the transmitted symbol by replacing $\mathrm{CH}_{1}$ and $\mathrm{CH}_{2}$ by the estimated ones. Thus, the two received symbols and the first symbol of each block of each antenna are used to estimated $\mathrm{CH}_{1}$ and $\mathrm{CH}_{2}$.

The estimated equation for $\mathrm{CH}_{1}$ and $\mathrm{CH}_{2}$ can be expressed as:

$$
\begin{aligned}
& \hat{C H}_{1}[n, k]=\frac{r[n, k] S_{1}^{*}[n, k]-r[n, k+1] S_{2}[n, k]}{\left|S_{1}[n, k]\right|^{2}+\left|S_{2}[n . k]\right|^{2}} \\
& \hat{C H}_{2}[n, k]=\frac{r[n, k] S_{2}^{*}[n, k]-r[n, k+1] S_{1}[n, k]}{\left|S_{1}[n, k]\right|^{2}+\left|S_{2}[n . k]\right|^{2}}
\end{aligned}
$$

Therefore simply by replacing $\mathrm{CH}_{1}$ and $\mathrm{CH}_{2}$ in equation (6) and (7) by $\hat{\mathrm{CH}_{1}}, \hat{\mathrm{CH}}_{2}$ in equation (8) and (9) it can be deduced :

$$
\hat{S_{i+2}}[n, k]=\hat{C H_{1}}[n, k] r[n, k+2]+\hat{C H} 2[n, k] r^{*}[n, k+3](10)
$$

$$
\hat{S_{i+3}}[n, k]=\hat{C H} 2[n, k] r[n, k+2]-\hat{C H} 1[n, k] r^{*}[n, k+3](11)
$$

Where $i=1,3,5 \ldots . . N-4$

The propose method is simple and cost, computation effective.

\section{PROPOSED CHANNEL EQUALIZATION METHOD}

The channel equalization is the most important part as it reduces the ISI affect and improve the BER of the system. In this paper the channel equalization is done using Scale Conjugate Gradient Method . SCG is used to train the feed forward neural network. The neural network used in our system Consist of three layers: input ,hidden and output. The number of nodes used in input layer are three, hidden Layer are ten and in the output layer the nodes are two. The transfer function used for input is Logistic Sigmoidal and for hidden layer is purelin. Linear is selected for the output. Figure 2 shows the model of the Neural Network Used for simulation.

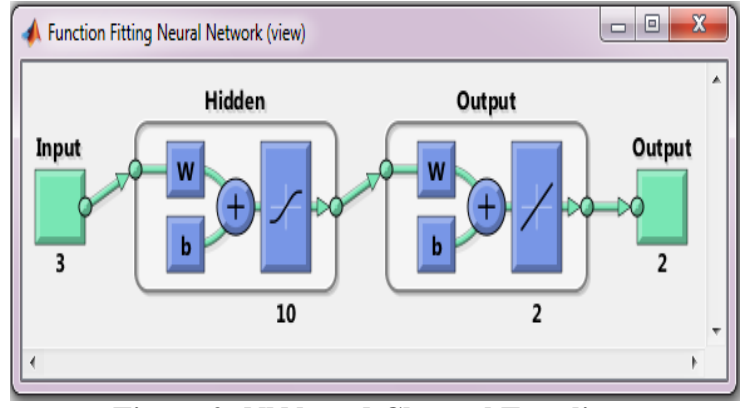

Figure 2: NN based Channel Equalizer

\section{SIMULATION RESULTS}

After the network is trained using the SCG method three parameters Such as channel length, signal length and channel noise are inputted to the network. For our simulation we have used signal length as 500, channel length as 20 and channel noise as 5. The simulation is done on MATLAB R2013a.Three different parameters Symbol Error Rate (SER),Bit Error Rate ( BER) and Minimum mean square error (MMSE) are plotted against different values of Signal to noise ratio ( SNR).

The results are tabulated in Table 2 and plotted in figure 3.

Table 2 : SNR VS BER/SER/MMSE

\begin{tabular}{|c|c|c|c|}
\hline SNR & BER & SER & MMSE \\
\hline 0 & 0.1519 & 0.5964 & 0.1484 \\
\hline 5 & 0.1078 & 0.1858 & 0.0769 \\
\hline 10 & 0.0488 & 0.0589 & 0.0377 \\
\hline 15 & 0.0181 & 0.0193 & 0.0188 \\
\hline 20 & 0.0061 & 0.0062 & 0.0054 \\
\hline 25 & 0.0022 & 0.0023 & 0.0021 \\
\hline 30 & 0.001 & 0.001 & 0.0006 \\
\hline 35 & 0.0006 & 0.0006 & 0.0005 \\
\hline 40 & 0.0005 & 0.0005 & 0.0003 \\
\hline
\end{tabular}

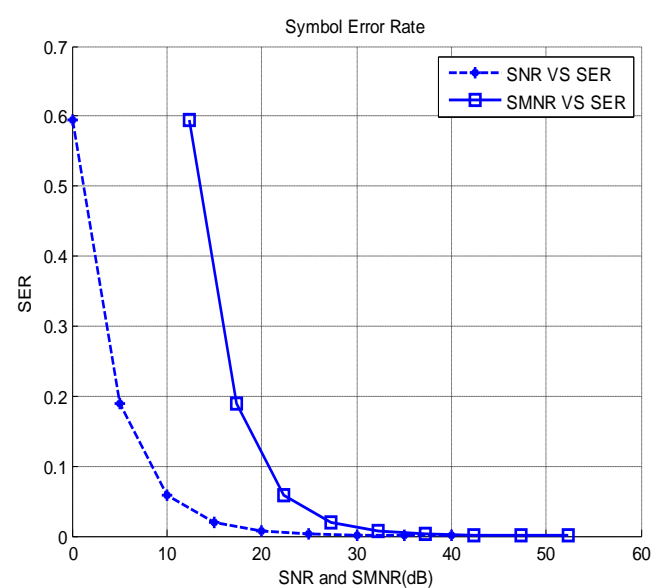

Graph 1. SNR/SNER VS SER

In Graph 1 the Symbol error rate (SER) are plotted against the two quantities signal to noise ratio (SNR) and Symbol to noise ratio (SMNR).Initially both behaves in the same manner but the slope of SMNR are more steep and both are coincide at the higher values. 


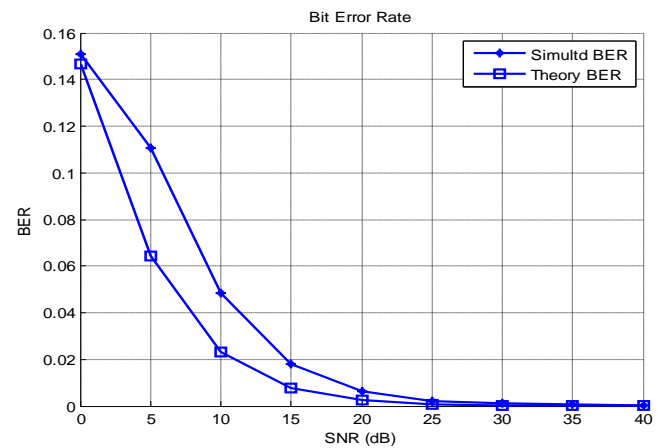

Graph 2. SNR VS BER

In graph 2 the BER at different values of SNR are plotted. It is clear from the graph that the proposed SCGNN algorithm are performing very good as the simulated BER are very close to theoretical BER at very low values of SNR and as the SNR are increased up to 25 the two response become coincide i.e. simulated result catches the theoretical values.

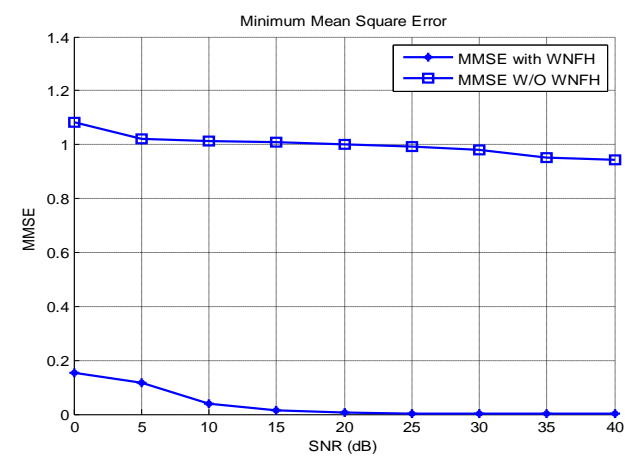

Graph 3. SNR VS MMSE

In Graph 3 we have plotted minimum mean square error (MMSE) with and without equalization. It is very clear that the MMSE with equalization are far better which shows the effectiveness of our proposed SCGNN algorithm for equalization.

\section{CONCLUSION}

Wireless transmission of digital signals through multipath frequency selective channels can be done in perfect way using OFDM technique. OFDM can mitigate distortion caused by frequency selective fast fading channels as in OFDM the same copy of the data message can be obtained at the receiving end each experience different fading conditions ( impossible to have deep fading at all the links) so the reception process can be performed in better manner as evident from graph 2 .

The performance of the STBC-OFDM receiver depends mainly on the channel equalization stage. It has proven that $\mathrm{NN}$ based equalizers are very effective in dealing this kind of problem which is true from the graph 3 .

\section{REFERENCES}

[1] Sanjana T and Suma M N, "Comparison of Channel

[2] Estimation and Equalization Techniques for OFDMSystems", Circuits and Systems: Aninternationaljournal (CSIJ), Vol 1,No 1, January 2014. Yi-Sheng Chen, "Semi-Blind Channel Estimation for

[3] MIMO Single Carrier With Frequency Domain Equalization Systems." IEEE Transaction on
VehicularTechnology, Vol 59.No 1, pp 53-63, January 2010.Delestre F and Sum Y, "Pilot Added Channel Estimation for MIMO-OFDM System", London Communication Symposium 2009.

[4] Osvaldo Simeone and Umberto Spagnolini, "Pilot Based Channel Estimation for OFDM Systems by Tracking the Delay Subspace", IEEE Transaction on Wireless Communications, vol. 3, NO 1, January 2004.

[5] Tian-Ming Ma,Yu-Song Shi and Tying-Guan Wang, “A Low Complexity MMSE for OFDM Systems Over Frequency Selective Fading Channels", IEEE Communication Letters, Vol. 16. No 3, March 2012.

[6] D. Gesbert, M. Shafi, S. Da-shan, P. J. Smith, and A. Naguib, "From theory to practice: an overview of MIMO space-time coded wireless systems," IEEE Journal on Selected Areas in Communications, vol. 21, pp. 281-302, 2003.

[7] Xiang Li, Wen-De Zhong, Arokiaswami Alphones and Changyuan $\mathrm{Yu}$, "Channel Equalization Using Independent

[8] Component Analysis in PDM-CO-OFDM",IEEE Photonics Technology Letters, $\mathrm{Vol}$ 26,No 5,pp 497 501,March 2014.W. Kirkland and K. Teo, "I/Q distortion correction for OFDM direct conversion receiver" Electron. Lett., vol. 39, pp. 131-133, 2003.

[9] L. Brotje, S. Vogeler, and K-D. Kammeyer, "Estimation and correction of transmitter-caused I/Q imbalance in OFDM systems," in Proc. 7thIntl. OFDM Workshop, , pp. 178-182. Sep. 2002

[10] Y. Egashira, Y. Tanabe, and K. Sato, "A novel IQ imbalance compensation method with pilot-signals for OFDM system," in Proc. IEEE VTC Fall, pp. 1-5,2006.

[11] A. Tarighat, R. Bagheri, and A. Sayed, "Compensation schemes and performance analysis of IQ imbalances in OFDM receivers," IEEE Trans. Signal Process., vol. 53, pp. 3257-3268, 2005.

[12] Saroj K. Meher, Ashish Ghosh*, B. Uma Shankar and Lorenzo Bruzzone, "Neuro-Fuzzy Fusion: A New Approach to Multiple Classifier System" , 9th IEEE International Conference on Information Technology (ICIT'06),2005.

[13] Ibnkahla,M, "Application for Neural Networks to Digital Communications-A survey", Signal Processing,80,pp 1185- 1215,2000

[14] S. M. Alamouti, "A simple transmit diversity technique for wireless communications," IEEE Journal on Selected Areas in Communications, vol. 16, pp. 1451-1458, 1998.

[15] Mehmat Kemal Ozdemir and Huseyin Arslan, "Channel Estimation For Wireless OFDM System", IEEE Communication Surveys,Vol.9,No.2 pp 18-42,2007.

\section{AUTHOR PROFILE}

Prof. Zainul Abdin Jaffery obtained his B. Tech and M. Tech. in Electronics and Communication Engineering from Aligarh Muslim University, Aligarh, India in 1987 and 1989 respectively. He obtained his $\mathrm{PhD}$ degree from Jamia Millia Islamia (a central Govt. of India University) in 2004. Dr. Jaffery started his carrier as lecturer at Jamia Millia Islamia in 1990. Presently he is working as Professor in the Department of Electrical Engineering, Faculty of Engineering and 
Technology, Jamia Millia Islamia, New Delhi, since 2010. Prof. Jaffery has published more than fifty research papers in the area of Electronics and communication engineering in refereed international /national journals and conferences. His research area includes applications of soft computing techniques in signal and image processing. Prof. Jaffery is a member of various academic societies of national and international repute

Zeeshan Ahmad Abbasi obtained his Bachelor's degree in Electronics and Communication with Honors from Jamia Millia Islamia, Delhi India. Then he obtained his Master's degree in Communication from Delhi College of Engineering, University of Delhi.He is pursuing his Ph.D from JMI Delhi. $\mathrm{He}$ has worked for 20 years in Police Communication, Ministry of Home affairs, Government of India on VSAT Networks and HF, VHF Communication. He has taught various Electronics and Communication Engineering papers at many reputed Universities like Jamia Millia Islamia, Hamdard University, Delhi University, Maharishi Dayanand University, IGNOU etc. 